\title{
K-Nearest Neighbor Classification Approach for Face and Fingerprint at Feature Level Fusion
}

\author{
Dhriti \\ PEC University of Technology \\ Chandigarh \\ India
}

\author{
Manvjeet Kaur \\ PEC University of Technology \\ Chandigarh \\ India
}

\begin{abstract}
Biometric system that based on single biometric called unimodal biometrics usually suffers from problems like imposter's attack or hacking, unacceptable error rate and low performance. So the need of using multimodal biometric system arises in such cases. The aim of this paper is to study the fusion at feature extraction level for face and fingerprint. The proposed system fuses the two traits at feature extraction level by first making the feature sets compatible for concatenation and then reducing the feature sets to handle the "problem of curse of dimensionality". After concatenation these features are classified. Features of both modalities are extracted using Gabor filter and Principal Component Analysis (PCA). K-Nearest Neighbour classifier is used to classify the different people in the database.

The experimental results reveal that the fusion of more than one biometric trait at feature level fusion with the K-Nearest Neighbor technique exhibits robust performance and increases its performance with utmost level of accuracy.
\end{abstract}

\section{Keywords}

Multimodal Biometrics, feature level fusion, Gabor filter, PCA, classifier.

\section{INTRODUCTION}

In recent years, biometrics authentication has seen considerable improvements in reliability and accuracy, with some of the traits offering good performance. However, even the best biometric traits to date are facing numerous problems. Thus a single biometric is not sufficient to meet the variety of requirements including matching performance imposed by several large-scale authentication systems.

Multi-biometric systems [1] remove some of the drawbacks of the uni-biometric systems by grouping the multiple sources of information. They address the problem of non-universality, since multiple traits provide sufficient population coverage. They also limit spoofing since it would be difficult for an impostor to spoof multiple biometric traits [2]. Ross and Jain [3] and A. Rattani et al. [4] discussed about various levels of fusion in multimodal biometric systems namely sensor level, feature level, match score level and decision level.

A. Sensor level: The raw data acquired from multiple sensors can be processed and integrated to generate new data from which features can be extracted. For example, in the case of fingerprint biometrics, the fingerprint image acquired from both optical and solid state sensors may be fused to generate a single image which could then be subjected to feature extraction and matching.

B. Feature level: Information extracted from the different sources is concatenated into a joint feature vector, which is then compared to an enrollment template (which itself is a joint feature vector stored in a database) and assigned a matching score as in a single biometric system.
C. Match score level: Feature vectors are created independently for each modality and are then compared to the enrollment templates which are stored separately for each biometric trait. Based on the proximity of feature vector and template, each subsystem computes its own matching score. These individual scores are finally combined into a total score, which is passed to the decision module.

D. Decision level: A separate authentication decision is made for each biometric trait. The decisions are then combined into a final vote. Fusion at the decision level is considered to be rigid due to the availability of limited information.

The Biometric system that integrates information at an earlier stage of processing is expected to provide more promising results than the systems that integrate information at later stage because of availability of more/ richer information. Since the feature set contains richer information about the input biometric data than the matching score or the output decision of a matcher, fusion at the feature level is expected to provide better recognition performance.

Fusion at the match score, rank and decision levels have been extensively studied in the literature. As early as 1993, Chibelushi et al. [5] proposed a scheme to integrate acoustic and visual speech (motion of visible articulators) for speaker recognition using a simple linear combination. B Duc et al. [5] proposed a simple averaging technique and compared it with the Bayesian integration scheme presented by Kittler et al. [6]. A multimodal person verification system is proposed, using three experts: frontal face, face profile, and voice. The best combination results are obtained for a simple sum rule. Hong and Jain in [7] discussed a multi-modal personal identification system which integrates two different biometrics i.e. face and fingerprints.

However, fusion at the feature level is a relatively understudied problem [4]. Ross and Govindarajan [8] discussed about the fusion of hand and face biometrics at feature extraction level. Fusion at this level is difficult to achieve in practice because multiple modalities may have incompatible feature set or the feature space may be unknown, concatenated feature vector may lead to the problem of curse of dimensionality, a more complex matcher may be required for concatenated feature vector and concatenated feature vector may contain noisy or redundant data thus leading to decrease in the performance of the classifier [8]

\section{FEATURE EXTRACTION METHOD FOR FACE AND FINGERPRINT}

The features of face and fingerprints are to be extracted before matching. Along with the application of pre-processing steps 
proper image registration is essential for a good recognition process. First, the original images have to be converted to the gray scale form. Then, some contrast and illumination adjustment operations are performed. All the images must be processed with the same illumination and contrast. Therefore, some histogram equalization operations are performed on these images, to obtain a satisfactory contrast. Also, the images are often corrupted by various types of noise, is to be filtered before matching. Gabor filter and Principal Component Analysis (PCA) are used in the approach.

A Gabor filter-based feature extraction is proposed. Some feature vectors which provide optimal characterization of the visual content of facial images. For this reason 2D Gabor filtering is chosen which is a widely used image processing tool, for feature extraction. A Gabor filter (Gabor Wavelet) represents a band-pass linear filter whose impulse response is defined by a harmonic function multiplied by a Gaussian function. Thus, a bidimentinal Gabor filter constitutes a complex sinusoidal plane of particular frequency and orientation modulated by a Gaussian envelope. [9]

Principal Component Analysis is a method used for feature extraction. Eigen faces approach is a Principal Component Analysis method, in which a small set of characteristic features

are used to describe the variations between face images. Assume that there are $p$ training images i.e. $i=1,2 \ldots p$. The aim is to compute feature vectors for the images in the training database. In order to apply PCA to the training set, a training data matrix A should be created which contains $\mathrm{p}$ rows. Then the covariance matrix of $A$ i.e. $C_{A}$ is generated out of it. Then the eigen values and eigen vectors of $\mathrm{C}_{\mathrm{A}}$ should be computed. The eigen vector images are called eigen face, since they are similar to human faces. Same calculations are done with the test images for finding out their feature vectors.

After feature extraction from these biometric traits the main aim is to fuse these feature vectors with simply applying concatenation approach which adds feature sets of two biometric modalities.

\section{FEATURE LEVEL FUSION}

\section{STRATEGY}

Feature level fusion is a simple concatenation of the feature sets obtained from different sources of information. Let $\mathrm{X}=$ $\left(\mathrm{x}_{1}, \mathrm{x}_{2},--------\mathrm{x}_{\mathrm{m}}\right)$ and $\mathrm{Y}=\left(\mathrm{y}_{1}, \mathrm{y}_{2},---------, \mathrm{y}_{\mathrm{n}}\right)$ denote feature vectors of face or fingerprint. Concatenation rule is applied on these feature vectors represented by $\mathrm{Z}$ vector, which would have better recognition capability of the individual. The vector $\mathrm{Z}$ is then input to the matcher which computes the proximity between two concatenated feature vectors. [10] Figure 1.1 shows the steps in feature level fusion.

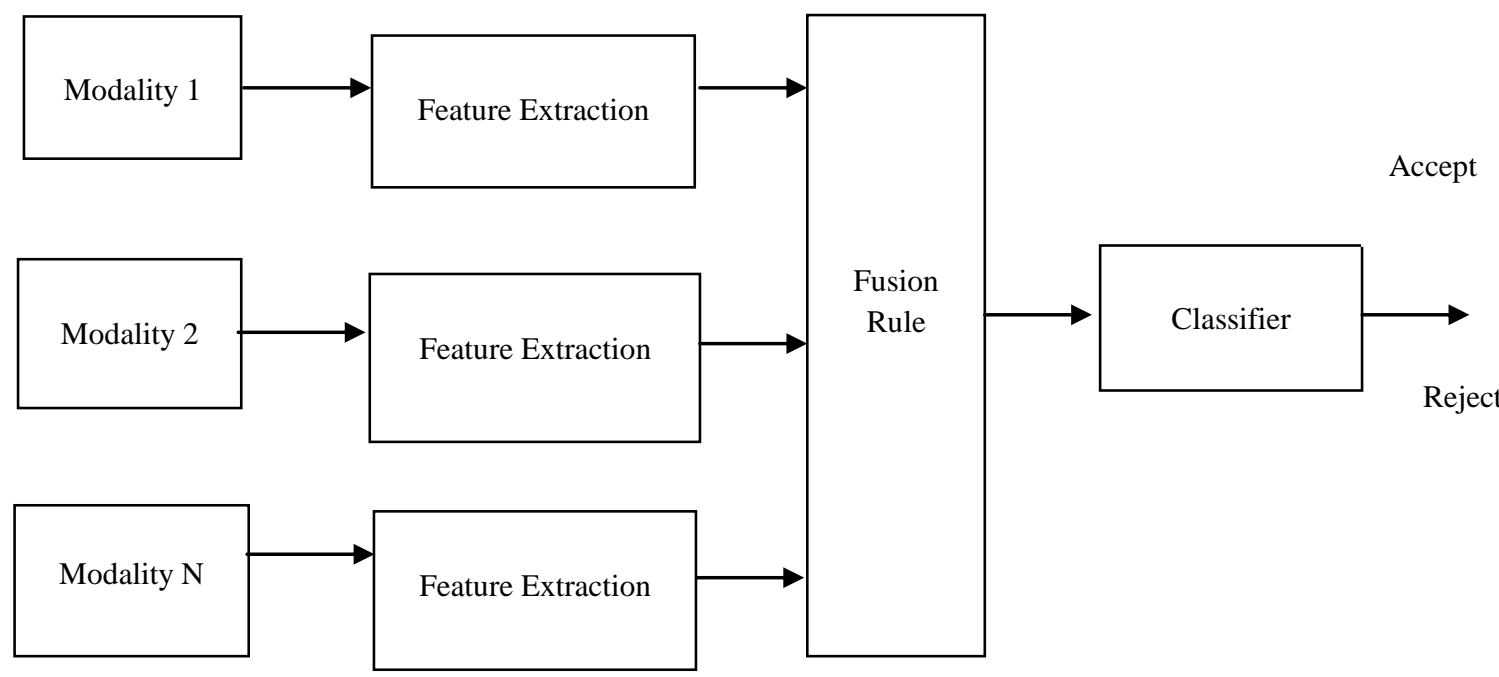

Fig 1: Feature Level Fusion 


\section{A. K-Nearest Neighbor}

$\mathrm{KNN}$ classifier is best suited for classifying persons based on their images due to its lesser execution time and better accuracy than other commonly used methods which include Hidden Markov Model and Kernel method. Although methods like SVM and Adaboost algorithms are proved to be more accurate than KNN classifier, KNN classifier has a faster execution time and is dominant than SVM. [11]

The simplest classification scheme is a nearest neighbor classification in the image space. Under this scheme an image in the test set is recognized by assigning to it the label of the closest point in the learning set, where distance are measured in image space.

The Euclidean distance metric [12] is often chosen to determine the closeness between the data points in KNN. A distance is assigned between all pixels in a dataset. Distance is defined as the Euclidean distance between two pixels. The Euclidean distance is given by:

$$
\mathrm{d}(\mathrm{x}, \mathrm{y})=\sqrt{\left(x_{1}-y_{1}\right)^{2} \ldots \ldots \ldots \cdot\left(x_{\mathrm{n}}-y_{n}\right.}
$$

This Euclidean distance is by default in a KNN classifier. But the distance between two features can be measured based on one of the distance cosine and correlation.

\section{B. K-NN Algorithm}

The $k$-nearest neighbour algorithm $(k-\mathrm{NN})$ is a method for classifying objects based on closest training examples in the feature space. K-NN is a type of instance-based learning, or lazy learning where the function is only approximated locally and all computation is deferred until classification. The k-nearest neighbour algorithm is amongst the simplest of all machine learning algorithms: an object is classified by a majority vote of its neighbours, with the object being assigned to the class most common amongst its $\mathrm{k}$ nearest neighbours ( $\mathrm{k}$ is a positive integer, typically small). If $\mathrm{k}=1$, then the object is simply assigned to the class of its nearest neighbour. [11]

1. Each data pixel value within the data set has a class label in the set, Class $=\{\mathrm{c} 1, \ldots, \mathrm{cn}\}$.

2. The data points', k-closest neighbors ( $\mathrm{k}$ being the number of neighbors) are then found by analyzing the distance matrix.

3. The k-closest data points are then analyzed to determine which class label is the most common among the set.

4. The most common class label is then assigned to the data point being analyzed.

\section{EXPERIMENTAL RESULTS}

The proposed approach has been tested on six different databases of face and fingerprint of different persons with PCA as a feature extraction tool and six databases of face and fingerprint with Gabor filter. The fingerprint images are taken from FVC databases [13].
The following procedure has been established for testing the mono-modal and multimodal algorithms:

Training: Two images per person is used for enrollment in the face and fingerprint verification system; for each individual. For these images we have to set their file format in jpg and the size of the face and fingerprint images should be same. The total number of images in the training database is 40.

Testing: The remaining images in the database per person are used for testing. For the multimodal testing, each client is tested against the first face and fingerprint samples of the rest of the users in the training database. The size and the file format should also be same as in the images in the training database.

Experiments were conducted in three sessions.

a) In the first experiment, face and fingerprint images are classified without fusion with the PCA as a feature extractor and check our model accuracy.

b) In the second experiment, classifications is applied on face and fingerprint images without fusion with the PCA as a feature extractor and check our model accuracy with KNN distance metrics like: City block, cosine and correlation.

c) In the third experiment, the classification of face and fingerprint is done with and without fusion of its feature vectors with all the distance metrics with Gabor filter as a feature extractor.

For all these experiments True Positives and False Negatives are calculated. From the presented results in table 1, it is evident that the introduction of fusion for face and fingerprint images increases the Model Accuracy (MA) at the feature extraction level. Model accuracy for this is calculated as:

Accuracy $=\frac{T P+T N}{P+N}$, where $T P$ is stands for True Positive and $T N$ stands for True Negative. $\mathrm{P}$ and $\mathrm{N}$ are the total of columns and $\mathrm{P}$ and $\mathrm{N}$ are calculated as: $\mathrm{P}=T P+F N$ and $N=F P+T N$

Table 1

\begin{tabular}{|c|c|c|c|}
\hline Sr. No & Modality & $\begin{array}{c}\text { MA without } \\
\text { fusion }\end{array}$ & $\begin{array}{c}\text { MA with } \\
\text { fusion (fafi) }\end{array}$ \\
\hline 1 & Face & 0.4 & \multirow{2}{*}{1.0} \\
\hline 2 & Fingerprint & 0.6 & \\
\hline
\end{tabular}

Fig. 2 shows that our model accuracy increases when we fuse its feature vectors. Table 1 shows that face (fa) gives $0.4 \%$ of model accuracy and fingerprint gives $0.6 \%$ but when we fuse both of them and classify with KNN our model accuracy increases upto $1.0 \%$ and it gives highest accuracy value with city block distance metric. 


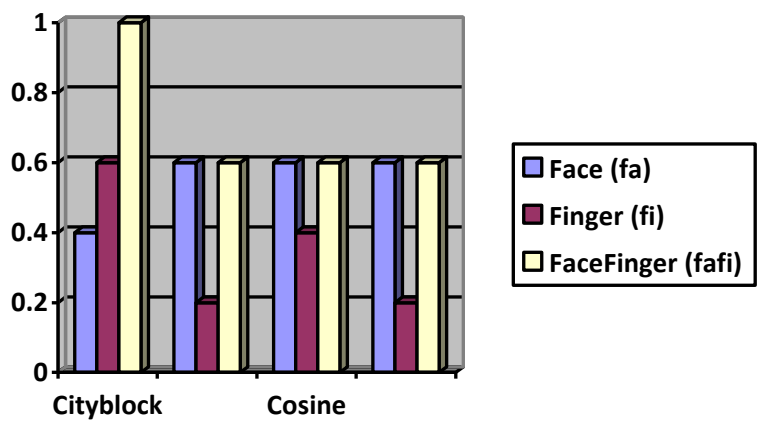

Fig 2: Performance analysis of system with fusion (PCA)

Table 2 shows the results about face and fingerprint with and without fusion. This test is performed on face and fingerprint images with Gabor filter as a feature extractor and $\mathrm{KNN}$ as a classifier.

Table 2

\begin{tabular}{|c|c|c|c|}
\hline Sr. No & Modality & $\begin{array}{c}\text { MA without } \\
\text { fusion }\end{array}$ & $\begin{array}{c}\text { MA with } \\
\text { fusion (fafi) }\end{array}$ \\
\hline 1 & Face & 0.67 & 1.0 \\
\hline 2 & Fingerprint & 0.67 & \\
\hline
\end{tabular}

Fig 3: Performance analysis of system with fusion (Gabor filter )

From our experiments True Positives for face and fingerprint and False Negative are calculated. The graphs showed below gives some conclusions about True Positives and False Negatives.

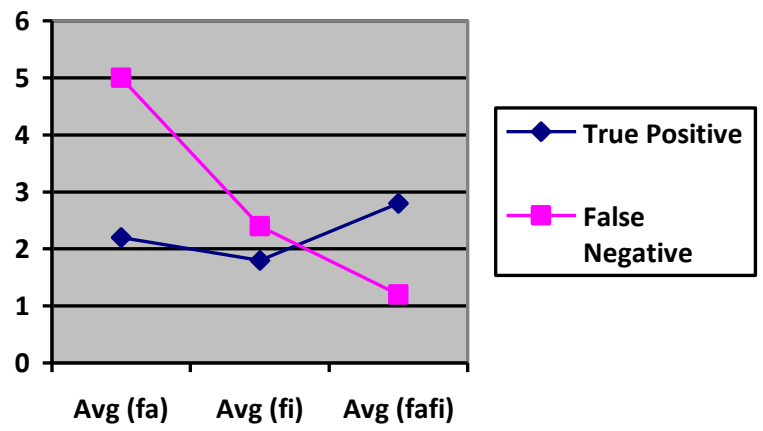

Fig 4: TP \& FP rate (with PCA)

In this graph the average of True Positives of face (fa), fingerprints (fi) and face and fingerprint both (fafi) are taken. The graph shows that the True Positive blue colored line is raising upwards, which means that if fusion is applied on biometric traits it increases the value of True Positives. For False Negative its value should decrease along the graph and the same is happening in this case. The false negative value is decreasing for face, fingerprint and when fusion is applied on both of these traits.
In Fig.5 we again calculate average of True Positives and False Negatives for face, fingerprint and both of them with Gabor filter. This is shown below:

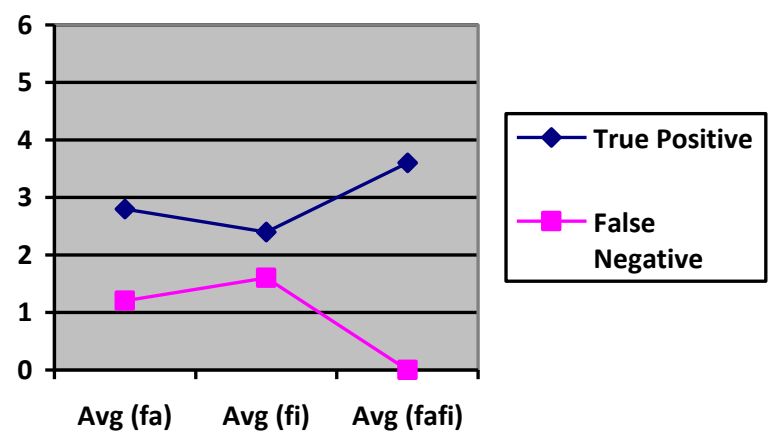

Fig 5: TP \& FP rate (with Gabor filter)

\section{CONCLUSION}

A multimodal biometric system based on the integration of face and a fingerprint trait was presented. These two traits are the most widely accepted biometric. In this paper a novel approach has been presented where both fingerprint and face images are processed with compatible feature extraction algorithms, fusion strategy is applied to both of the biometric traits and data is classified to obtain the increased model accuracy.

The performance of both face and fingerprint with and without fusion were compared at feature extraction level. Interestingly the fusion at the feature level provides better results as compare to test these modalities individually. Here the experiments also find that KNN classifier with city block distance metric gives the highest fusion value as compare to Euclidean, cosine and correlation.

\section{REFERENCES}

[1] L. Hong, Anil K Jain, "Can multi-biometric improve performance". IEEE Workshop on Automatic Identification Advanced Technologies, pp 59-64, Oct. 1999.

[2] A.k .Jain and A. Ross, "Multibiometric systems, Communications of the ACM, 47 (1), pp. 34-40, 2004.

[3] A. Ross and A.K. Jain, "Information Fusion in Biometrics", Pattern Recognition Letters, 24, pp. 21152125, 2003.

[4] A. Rattani, D. R. Kisku and M. Bicego, "Feature level fusion Of face and fingerprint Biometrics", IEEE, pp 1-6, Sept. 2007.

[5] Benoit. Duc, G. Maitre, S. Fischer, and J. Bigun, "Person authentication by fusing face and speech information", In Proc. of the First International Conference on Audio and Video-based Biometric Person Authentication, Lecture Notes in Computer Science. Springer Verlag, pp 311-318, 1999

[6] J. Kittler, M. Hatef, R. P. W. Duin, and J. Matas, "On combining classifiers", IEEE Transactions on Pattern 
Analysis and Machine Intelligence, pp.

226-239, March 1998

[7] L. Hong and A. Jain, "Integrating Faces and Fingerprints for Personal Identification", IEEE Transactions

on Pattern Analysis and Machine Intelligence, pp. 12951307, December 1998

[8] A. Ross and R. Govindarajan, "Feature Level Fusion Using Hand and Face Biometrics", Proc. of SPIE Conference on Biometric Technology for Human Identification II, Orlando, USA, pp. 196-204, March 2005 .

[9] Tudor BARBU, "Gabor Filter-Based face recognition technique", proceedings of the Romanian Academy, Series A, Volume 11, pp. 277-283, Nov. 2010.

[10] Ajita Rattani, D.R Kisku, mamuele bicego and Massimo Tistarelli, "Robust feature level Multibiomeric
Classification”, IEEE, Biometric Symposium, pp 1-6, Aug. 2006.

[11] Vaidehi, S.Vasuhi et.al, "Person authentication using face detection", Proceedings of the World Congress on Engineering and Computer Science, pp 222-224, 2008.

[12] M. A. Turk and A. P. Pentland, "Face recognition using eigenfaces", IEEE Computer Society Conference on Computer Vision and Pattern Recognition, Proceedings CVPR , pp 586-591, Jun. 1991.

[13] D. Maio, D. Maltoni, R. Cappelli, J. L. Wayman and A. K. Jain,"FVC2001-2004: Fingerprint Verification Competition", in proceedings International Conference on Biometric Authentication (ICBA04), Hong Kong, pp. 1-7, July 2004. 